\title{
Isolation and molecular characterization of recombinant Echinococcus granulosus P29 protein (recP29) and its assessment for the post-surgical serological follow-up of human cystic echinococcosis in young patients
}

\author{
Nadia Ben Nouir ${ }^{a, b}$, Christian Gianinazzi ${ }^{b}$, Mohamed Gorcii ${ }^{a}$, \\ Norbert Müller ${ }^{b}$, Abdellatif Nouri ${ }^{c}$, Hamouda Babba ${ }^{a}$, Bruno Gottstein ${ }^{b, *}$
}

a University of Monastir, Faculty of Pharmacy, Department of Clinical Biology B, Laboratory of Parasitology and Mycology, 99UR/08-05 1-rue avicenne, 5000 Monastir, Tunisia

b Institute of Parasitology, University of Bern, Laengassstrasse 122, CH-3012 Bern, Switzerland

c E.P.S. Fattouma Bourguiba, Pediatric Surgery Service, 5000 Monastir, Tunisia

Received 28 May 2008; received in revised form 30 September 2008; accepted 30 September 2008 Available online 21 November 2008

\section{KEYWORDS \\ Cystic echinococcosis; \\ Echinococcus \\ granulosus; \\ Recombinant \\ proteins; \\ P29 antigen; \\ ELISA; \\ Immunoblotting}

\begin{abstract}
Summary We synthesized recombinant Echinococcus granulosus protoscolex recP29 antigen to be preliminarily assessed by ELISA and immunoblotting. RecP29-serology was carried out on 54 young patients with cystic echinococcosis (CE). Patients were classified into either cured (CCE) $(n=40)$ or non-cured (NCCE) $(n=14)$ CE patients. RecP29 ELISA showed a gradual decrease of antibody concentrations in all CCE cases that were initially (before treatment) seropositive to this antigen ( 25 out of 40 ) or that seroconverted following treatment. A complete seronegativity was reached within 3 years post-surgery in all of these cases. Conventional HCF ELISA yielded seronegativity in only $10 \%$ of initially recP29-seropositive CCE patients $(P=0.086)$. Likewise, recP29 immunoblotting yielded seronegativity in $93 \%$ of 29 out of 40 initially recP29-immunoblot-positive CCE patients after 3 years follow-up, compared with $72 \%$ in the HCF immunoblotting $(P=0.060)$. Eleven out of 14 NCCE patients were initially positive by recP29 ELISA, and 10 out of these maintained a marked anti-recP29 antibody reactivity until the endpoint of the follow-up period. All 14 NCCE cases were initially seropositive by recP29 immunoblotting, and 13 cases remained seropositive until the end of the study. Thus, recombinant P29 protein appears prognostically useful for monitoring those post-surgical CE cases with an initial seropositivity to this marker.

(c) 2008 Royal Society of Tropical Medicine and Hygiene. Published by Elsevier Ltd. All rights reserved.
\end{abstract}

* Corresponding author. Present address: Institute of Parasitology, Laengassstrasse 122, CH-3001, Bern, Switzerland.

Tel.: +413163124 18; fax: +41316312477.

E-mail address: bruno.gottstein@ipa.unibe.ch (B. Gottstein).

0035-9203/\$ - see front matter ๑ 2008 Royal Society of Tropical Medicine and Hygiene. Published by Elsevier Ltd. All rights reserved. doi:10.1016/j.trstmh.2008.09.020 


\section{Introduction}

One of the particular features characterizing the followup period of treated cases of cystic echinococcosis (CE) is recurrence or relapse after treatment, e.g. as a consequence of non-radical surgical procedures or peri-surgical spillage of parasite material, especially protoscoleces. ${ }^{1}$ Consequently, an appropriate strategy and methodology is mandatory in follow-up of CE cases, especially in view to reliably evaluating the success or failure of therapy, and detecting newly growing or relapsing cysts as early as possible. In this respect, imaging techniques such as X-ray, ultrasonography (US), computed tomography (CT) and magnetic resonance imaging (MRI) are very useful, although sometimes a concise discrimination between inactivated/dead or still viable cysts cannot be provided with these techniques. ${ }^{2}$ Immunodiagnosis has been used many times to support clinical follow-up of CE patients after surgical or pharmacological treatment,,$^{3-8}$ but the researchers of most of these studies agreed that an improvement of the serological tools may be of great benefit.

So far, predominantly crude antigens have been used as the main antigen source for carrying out primary serodiagnosis and also serological follow-up studies on patients after surgical or pharmacological treatment. ${ }^{9}$ Post-treatment monitoring of $\mathrm{CE}$ patients with such antigens has been of only limited help in prognostically classifying the status of a CE patient after treatment, and serological results allowed the discrimination of cured and non-cured status of CE only retrospectively. This could be due to the temporally delayed reaction of antibody concentrations to clinical changes, ${ }^{10}$ in addition to the persistence of specific antibodies for several years after recovery. ${ }^{1,11,12}$ Importantly, this made it difficult to predict early enough the outcome of surgery. Therefore, new markers that allow the prognostic assessment of post-operative development of disease are highly demanded. Considerable efforts have recently been undertaken to develop recombinant proteins with potential usefulness in serodiagnosis. Thus, several recombinant antigens have been expressed and serologically evaluated, including the two different $A g B$ subunits EgAgB1 and EgAgB2, ${ }^{13,14}$ and recombinant Ag5, ${ }^{15}$ recEpC $1^{16}$ and recEgCMDH (cytosolic malate dehydrogenase homologue). ${ }^{17}$ Most of these studies preliminarily documented a high diagnostic performance of the recombinant antigens. ${ }^{9,14-16,18,19}$ Alternatively, synthetic peptides including $\mathrm{p} 176,{ }^{20,21}$ derived from the antigen $B$ sequence, and the 89-122, which mimics an epitope of $\mathrm{Ag} 5,{ }^{22}$ also provided interesting findings. Unfortunately, these recombinant antigens and synthetic peptides were not (or only poorly) investigated for their prognostic value in the serological follow-up of CE.

We recently reported an interesting double band of $27-28 \mathrm{kDa}$, which remained persistently recognized by active or non-cured CE (NCCE). ${ }^{23}$ Conversely, cured CE patients (CCE) progressively lost their antibody levels to this $27-28 \mathrm{kDa}$ double band. ${ }^{23}$

The objective of the present study was to clone and express the gene(s) encoding for this/these proteins and to provide a preliminary assessment of the serological followup value of this antigenic tool.

\section{Materials and methods}

\subsection{Antigens, MS-MS and database search}

Protoscolex soluble protein extract (PSSA) was obtained and processed as previously described. ${ }^{23}$ Proteins $(10 \mu \mathrm{g} / \mathrm{cm}$ gel slot) were subsequently separated on a 5-20\% linear gradient polyacrylamide gel as described by Gottstein et al. ${ }^{24}$ Proteins were visualized using a silver stain protocol specific for mass spectrometry as described by Shevchenko et al. ${ }^{25}$ Protein bands of interest were excised from the SDSpolyacrylamide gel, and subjected to proteomic analysis at Core Facility Proteomics, Centre Medical Universitaire, Geneva University, Switzerland. Briefly, bands were subjected to in-gel trypsin digestion. The obtained fragments were separated and analyzed by liquid chromatography (LC) coupled to an electrospray mass spectrometer (LCESI-MS/MS). The resulting spectra were searched against the National Center for Biotechnology Information nonredundant and expressed sequence tag (EST) databases by using the MASCOT search engine (MATRIX Science, London, UK). MS data were extracted using the Mascot script within Analyst QS (http://www.matrixscience.com) and the databases were searched in the tandem MS (MS/MS) ion mode. Hits to a number of contiguous overlapping ESTs were obtained, and these were aligned to obtain the final sequence. The deduced amino acid sequence was investigated for potential phosphorylation and glycosylation sites by using the following ExPASy proteomics servers: NetPhos, NetNGlyc, NetOGlyc and Yin OYang (http: / / www.expasy.ch). In addition, sequence homology search (quick Blast $P$ engine at ExPASy proteomics server and MultAlin at the Institut National de Recherche Agronomique, Paris, France), $\mathrm{N}$-terminal signal peptide analysis (Signal $\mathrm{P} 3.0$ program; http://www.expasy.ch) and identification of specific domain (SMART: simple modular architecture research tool at EMBL, Heidelberg, Germany; http://www.smart.emblheidelberg.de/smart/show_motifs) were also performed.

\subsection{Parasite RNA extraction and RT-PCR}

Total RNA was prepared from E. granulosus protoscoleces (G1 strain) freshly isolated from a sheep hydatid cyst using the RNeasy Mini Kit 50 (QIAGEN, Basel, Switzerland; cat. no. 74104) according to the manufacturer's instructions. cDNA synthesis was performed using Omniscript Reverse Transcriptase (QIAGEN; cat. no. 205111). Briefly, cDNA was synthesized with total RNA at a final reaction volume of $20 \mu \mathrm{l}$ containing $2 \mu \mathrm{l}$ of $10 \times$ buffer RT, $0.5 \mathrm{mmol} / \mathrm{l}$ of each dNTP, $1 \mu \mathrm{mol} / /$ of Random primers (Promega, Duebendorf, Switzerland), $10 \mathrm{U}$ RNase inhibitor and $4 \mathrm{U}$ Omniscript Reverse Transcriptase. Following incubation for $1 \mathrm{~h}$ at $37^{\circ} \mathrm{C}$, Omniscript Reverse Transcriptase was inactivated by heating the mixture to $93^{\circ} \mathrm{C}$ for $5 \mathrm{~min}$.

\subsection{PCR and cloning}

Synthesized cDNA used for the production of the recombinant protein was amplified using specific primers designed from published nucleotide sequences (see Table 1). CACC 
Table 1 Primer pairs used for amplification of P29 gene sequences ${ }^{\mathrm{a}}$.

\begin{tabular}{ll}
\hline Forward 1 (F1): & 5'-CACCTCCGGATTTGACGTTACTAAG-3' \\
Forward 2 (F2): & 5'-CACCACCGACAAACTGGGTACTGC-3' \\
Reverse (R): & 5'-CTACTCGCCCAGCATCATCATACT-3'
\end{tabular}

a These primers were designed based on nucleotide sequence information available in GenBank (accession no. AF078931). Primers were synthesized by MWG Biotechnologies, Basel, Switzerland.

nucleotides were added to the $5^{\prime}$ end of the forward primer to facilitate cloning of PCR products. PCR amplification was performed in a $50 \mu \mathrm{l}$ reaction mixture containing $1 \times$ PCR buffer $\left(2 \mathrm{mmol} / / \mathrm{MgSO}_{4}\right), 0.2 \mathrm{mmol} / \mathrm{l}$ dNTP mix, 1 pmole of each primer and 1.5 U pfu DNA polymerase. Samples were amplified in a Gene Amp PCR system 9700 (Applied Biosystems, Rotkreuz, Switzerland) thermal cycler using the following temperature profile: denaturation, $94^{\circ} \mathrm{C}, 30 \mathrm{~s}$ (first cycle 2 min at $94^{\circ} \mathrm{C}$ ); annealing, $52^{\circ} \mathrm{C}, 30 \mathrm{~s}$; extension, $72{ }^{\circ} \mathrm{C}$, $3 \mathrm{~min}$. Following 40 cycles, a final extension step was added for 5 min at $72^{\circ} \mathrm{C}$. PCR products were then subcloned into the PET 151/D-TOPO vector (Invitrogen, Carlsbad, CA, USA; cat. no. $\mathrm{K}$ 151-01), using the polylinker of the vector designed to produce proteins with an $\mathrm{N}$-terminal $6 \times$ His tag. The recombinant vector was used to transform $E$. coli one-shot TOPO10 chemically competent cells. Transformants were selected on LB agar plates containing $100 \mu \mathrm{g} / \mathrm{ml}$ ampicillin after overnight culture at $37^{\circ} \mathrm{C}$.

\subsection{Colony PCR}

Colony PCR was used to check whether a specific colony had the desired recombinant plasmid. Small colonies were suspended in $50 \mu \mathrm{l} \mathrm{H}_{2} \mathrm{O}$, heated for $5 \mathrm{~min}$ at $95^{\circ} \mathrm{C}$ and then amplified by PCR as follows. PCR was performed in a $100 \mu \mathrm{l}$ reaction volume using $1 \mu \mathrm{l}$ colony suspension, containing $1 \times$ PCR buffer $\left(1.5 \mathrm{mmol} / / \mathrm{MgCl}_{2}\right)$, 1 pmole of each primer (T7 forward primer/insert reverse primer or insert forward/T7reverse), $0.1 \mathrm{mmol} / \mathrm{l} \mathrm{dNTP} \operatorname{mix}$ and $2.5 \mathrm{U}$ Taq polymerase. Samples were amplified in a thermal cycler using the following temperature profile: denaturation, $94^{\circ} \mathrm{C}$ (first cycle $2 \mathrm{~min}$ at $94^{\circ} \mathrm{C}$ ) $30 \mathrm{~s}$; annealing $55^{\circ} \mathrm{C}, 30 \mathrm{~s}$; extension, $72^{\circ} \mathrm{C}, 1 \mathrm{~min}, 30 \mathrm{~s}$. Following 40 cycles, a final extension step was added for $5 \mathrm{~min}$ at $72{ }^{\circ} \mathrm{C}$.

\subsection{Expression and purification of recP29}

Positive clones picked from LB agar plates were cultured in $5 \mathrm{ml}$ LB medium with $100 \mu \mathrm{g} / \mu \mathrm{l}$ ampicillin and subjected for plasmid extraction using the Plasmid Miniprep Kit (peqLab, Erlangen, Germany). Plasmids were then transfected into $E$. coli BL 21 (DE3), chemically competent cells, and incubated in $250 \mu \mathrm{l} \mathrm{SOC}$ medium with shaking for $40 \mathrm{~min}$ at $37^{\circ} \mathrm{C}$, followed by overnight cultivation at $37^{\circ} \mathrm{C}$ on LB amp agar plates. Two hundred microlitres of overnight culture was inoculated into a new LB medium containing the appropriate antibiotic, then submitted to mass production using $5 \mathrm{ml}$ overnight culture inoculated into $200 \mathrm{ml}$ LB amp medium with $2 \mathrm{~h}$ incubation and shaking at $37^{\circ} \mathrm{C}$ until an optical den- sity of $0.5-0.8$ was reached. Isopropyl- $\beta$-D-thiogalactoside (IPTG; Sigma, Buchs, Switzerland) was subsequently added to a final concentration of $1 \mathrm{mmol} / \mathrm{l}$. Expressed recombinant protein was extracted from bacterial cells using Triton X-100 buffer followed by four sonications of $30 \mathrm{~s}$ at $57 \mathrm{~W}$ in a sonifier cell disruptor B-12 (Bransonic Power Company, Danbury, CT, USA), and subsequently analysed for its solubility. After centrifugation $\left(14000 \mathrm{~g}\right.$ for $10 \mathrm{~min}$ at $\left.4{ }^{\circ} \mathrm{C}\right)$, the clarified supernatant was loaded into a cobalt-based resin column (TALON HIS-Tag Purification resin; BD Biosciences, Allschwil, Switzerland) to capture the His-tagged recombinant protein. After three times washing with PBS $50 \mathrm{mmol} / \mathrm{l}, 5 \mathrm{mmol} / \mathrm{l}$ imidazole $\mathrm{pH} 7.0$, the recombinant fusion protein was eluted using buffered imidazole at $150 \mathrm{mmol} / \mathrm{l}$. Eluted protein was dialyzed overnight against PBS and stored at $-20^{\circ} \mathrm{C}$ until used. Protein expression and purification were checked by SDS-PAGE and Coomassie Blue R-250 staining. Protein concentration was determined using the Bio-Rad-Bradford protein assay (Bio-Rad Laboratories AG, Reinach, Switzerland; cat. no. 500-0002) with bovine plasma immunoglobulin as standard.

\subsection{Sera from CE patients}

In total, 290 sera were collected from 54 patients aged between 3 and 15 years (Table 2). All cases were surgically, radiologically and/or histologically proven. For each case, a first serum specimen was obtained at the time of diagnosis (before medical intervention) and further samples during a follow-up period ranging between $7 \mathrm{~d}$ and 5 years post-surgery and/or chemotherapy. The intervals and numbers of follow-up steps were variable between the patients, and the mean serum sample number per patient was $7 \pm 2$. Basically, sera were the same as those used in our two previous investigations, ${ }^{10,23}$ thus allowing a direct comparative assessment. Briefly, the follow-up design was as follows: subsequent to the initial diagnostic investigation, each patient was subjected to both serology and imaging investigation 1 week after surgery and/or chemotherapy, and then after 1 month, after 6 months and once the following year. Hydatid cysts were classified into five types according to $\mathrm{WHO}^{26}$ classification: type CE 1 (unilocular, simple cysts); type CE 2 (multivesicular, multiseptated cysts); type CE 3 (uniloclar cysts, which may contain daughter cysts); type CE 4 (heterogeneous or hyperechoic degenerative contents); and type CE 5 (calcified cysts). Types CE1 and CE 2 represent the active and progressive phase of CE; type CE 3 represents the transitional stage, during which it usually starts to degenerate; and types CE 4 and CE 5 represent the inactive and regressive phase. Depending on imaging findings at the endpoint of follow-up and on the respective surgeon's classification, 54 patients were clustered into two different groups: group A included cured or inactive hydatid disease cases (CCE; $n=40 / 54$ ), in which the hydatid cyst had disappeared following surgery or had fully calcified in five cases (type CE 5); the other cases remained inactive for the whole study period with regard to clinical symptoms, size and shape of remaining lesions. Group $B$ included patients who presented still active CE (NCCE; $n=14 / 54$ ) and/or a progressive form of disease after obviously unsuccessful (non-complete) surgical removal of the 
Table 2 Overview of the clinical features of the two groups of young cystic echinococcosis ${ }^{\mathrm{a}}$ patients included in the present study.

\begin{tabular}{|c|c|c|c|c|c|c|c|c|c|c|c|c|c|c|c|}
\hline \multirow[t]{2}{*}{$n^{\mathrm{b}}$} & \multicolumn{2}{|c|}{ Sex } & \multirow{2}{*}{$\begin{array}{l}\text { Mean age (range) } \\
\text { in years }\end{array}$} & \multicolumn{6}{|c|}{ Cyst localization and type ${ }^{c}$} & \multicolumn{6}{|c|}{ Therapy and follow-up ${ }^{e}$} \\
\hline & $\mathrm{F}$ & M & & Lun & & Live & & Mul & iple ${ }^{d}$ & $n^{f}$ & Period & Surgery & ABZ & Surgery & Years \\
\hline CCE 40 & 16 & 24 & $9(3-15)$ & 18 & $\begin{array}{l}\text { CE1 } \\
(18)\end{array}$ & 11 & $\begin{array}{l}\text { CE1 (5) } \\
\text { CE2 (2) } \\
\text { CE3 (3) } \\
\text { CE4 (1) }\end{array}$ & 11 & $\begin{array}{l}\text { CE1 (9) } \\
\text { CE2 } \\
(2)\end{array}$ & 6 & $2-48$ & 34 & 0 & 6 & $3 \pm 2$ \\
\hline NCCE14 & 4 & 10 & $9(4-14)$ & 5 & $\begin{array}{l}\text { CE1 } \\
\text { (5) }\end{array}$ & 6 & $\begin{array}{l}\text { CE1 (2) } \\
\text { CE2 (2) } \\
\text { CE3 (2) }\end{array}$ & 3 & $\begin{array}{l}\text { CE1 (2) } \\
\text { CE3 } \\
(1)\end{array}$ & 9 & $12-60$ & 5 & 2 & 7 & $3 \pm 2$ \\
\hline
\end{tabular}

a Cured cystic echinococcosis (CCE) and non-cured cystic echinococcosis (NCCE) patients.

b Total number of patients.

c Cyst type (CE1 to CE4; no CE5) corresponds to the sonographic classification carried out at initial diagnosis, according to WHO26.

d Multiple locations indicate that cysts were detected in the liver and the lungs (10 cases), the liver and spleen (one case), the liver and mediastinum (one case) or the liver, lungs and heart (two cases).

e For CCE patients, chemotherapy [albendazole (ABZ)] was added to surgery when cysts were considered as fissured. In NCCE patients, ABZ was administered in cases exhibiting fissured cysts, multiple cysts and disseminated or inoperable cysts left after surgery. Cysts were considered to be fissured when the laminated layer and consequently germinal layer presented visible damages that no longer maintained the original healthy status of hydatid cysts. If respective cysts were punctured, the hydatid fluid appeared milky or coloured, and high protein concentrations (i.e. $\geq 1 \mathrm{mg}$ protein $/ \mathrm{ml}$ ) were resolved.

${ }^{f}$ Number of patients who received chemotherapy (ABZ), alone or combined with surgery. 
cyst(s), or for whom surgery was not feasible (type CE 1, CE

2 and CE 3; for more details see Ben Nouir et al. ${ }^{10,23}$ ).

Sera were tested by ELISA and immunoblotting altogether in one run in order to avoid temporally related inter-test variations.

\subsection{SDS polyacrylamide gel electrophoresis (SDS-PAGE) and immunoblotting}

Recombinant P29 protein was solubilized with SDS sample buffer (1 mol/l Tris $\mathrm{HCl}, \mathrm{pH} 6.8$ containing 2\% SDS, 5\% 2 - $\beta$-mercaptoethanol and $10 \%$ glycerol) at $100{ }^{\circ} \mathrm{C}$ for $5 \mathrm{~min}$ and electrophoretically separated in a $15 \%$ polyacrylamide gel. Recombinant protein was loaded at a concentration of $0.2 \mu \mathrm{g} / \mathrm{cm}$ slot of the preparative gel. HCF was electrophoresed as previously described. ${ }^{10}$ Electrophoretically resolved molecules were transferred onto nitrocellulose (MB 85) and then cut into strips of $3 \mathrm{~mm}$ width. Strips were subsequently incubated with sera diluted $1 / 100$ in PBS plus $5 \%$ skimmed milk and $0.3 \%$ Tween 20 . Incubation was overnight at $4{ }^{\circ} \mathrm{C}$. Strips were then washed and incubated with goat anti-human IgG horseradish peroxidase conjugate diluted 1/400 (Sigma A6029) for $2 \mathrm{~h}$ at room temperature. Colour reaction was developed using the chromogenic substrate 3,3'-diaminobenzidine tetrahydrochloride (DAB) and $0.03 \% \circ \mathrm{H}_{2} \mathrm{O}_{2}$.

\subsection{ELISA}

Nunc Immulon ELISA microplate strips were coated by overnight incubation at $4{ }^{\circ} \mathrm{C}$ with $50 \mu$ l per well of either recombinant protein $(1.0 \mu \mathrm{g} / \mathrm{ml})$, or $\mathrm{HCF}(10.0 \mu \mathrm{g} / \mathrm{ml})$ diluted in ELISA coating buffer $\left(0.1 \mathrm{~mol} / \mathrm{l} \mathrm{NaHCO}_{3} / \mathrm{Na}_{2} \mathrm{CO}_{3}\right.$ $\mathrm{pH} 9.6$, and containing $0.02 \% \mathrm{NaN}_{3}$ ). The wells were then washed three times with washing buffer (PBS, 3\% Tween 20) and subsequently blocked with blocking buffer, consisting of PBS ( $\mathrm{pH} 7.2$ ) containing $0.05 \%$ bovine haemoglobin and $0.02 \% \mathrm{NaN}_{3}$. Sera were diluted 1:100 in blocking buffer, and phosphatase alkaline-labelled anti-human IgG conjugate (Sigma, A 5403) was used at a 1:2500 dilution. All incubations were carried out at $37^{\circ} \mathrm{C}$ for $30 \mathrm{~min}$. Plates were finally washed four times. After incubation with the substrate for $15 \mathrm{~min}$, the reaction was stopped with $10 \mu \mathrm{l} 3 \mathrm{~N} \mathrm{NaOH}$. The absorbances $\left(\mathrm{A}_{405} \mathrm{~nm}\right)$ were read with a microplate reader (Dynex Technologies BioConcept, Allschwil, Switzerland) and the results expressed in arbitrarily defined antibody units (AU) as previously described. ${ }^{27}$

\subsection{Statistical analyses}

Student's $t$ test was used to calculate the $\chi^{2}$ performance for comparing seropositivity between each group of patients with each type of antigen. A $P$-value of $\leq 0.05$ was considered statistically significant.

\section{Results}

\subsection{MS-MS analysis and database search}

The resulting peptide fragments from each excised band of 27 and $28 \mathrm{kDa}$ were individually subjected to database research homology using the Mascot research engine. On the one hand, overlapping fragment sequences resulting from the $27 \mathrm{kDa}$ band revealed $51 \%$ sequence coverage of amino acid sequences corresponding to the P29 E. granulosus protein (SwissProt accession no. Q9U8G7), and 10\% sequence coverage of that corresponding to Taenia solium triosephosphate isomerase protein (SwissProt accession no. Q9GTX8). These proteins were identified with high confidence. On the other hand, overlapping peptide fragments resulting from the $28 \mathrm{kDa}$ band showed $11 \%$ coverage sequence of actin amino acid sequence of $T$. solium (SwissProt accession no. P68555), with low confidence, and $37 \%$ sequence coverage of the 14-3-3 homologue two amino acid sequence of $E$. granulosus (SwissProt accession no. Q8MUA4) and 23\% of that of the putative 14-3-3 protein of $E$. granulosus (SwissProt accession no. Q56J98).

Based on these findings and our previous experiences with $E$. multilocularis $14-3-3^{28}$ and a publication on $\mathrm{P} 29,{ }^{29}$ we decided to carry on further experiments only with P29. $\mathrm{P} 29$ is a metacestode-specific component of $E$. granulosus ${ }^{29}$ with a corresponding amino acid sequence predicted from the open reading frame composed of 238 residues, with a deduced molecular mass of $27097 \mathrm{Da}$ and a predicted isoelectric point of 5.6. This protein contains 1 BAR domain from position 18 to 238 amino acids and does not contain a signal peptide cleavage site (signal $P$ 3.0). The deduced P29 sequence contains 19 sites for potential serine (10), threonine (6) and tyrosine (3) phosphorylations (NetPhos 2.0) and two sites for potential asparagine-threonine $\mathrm{N}$ glycosylations (NetNglyc1.0), in addition to four sites for potential serine-threonine $\mathrm{N}$ acetylation (Yin OYang 1.2).

Homology searches in the SwissProt database using the quick Blast P engine showed that P29 has a full identity to the Antigen 'S' epitope (Fragment) of Echinococcus granulosus ${ }^{30}$ and 97\% identity to antigen 6 (Fragment) [Adama6] of Echinococcus multilocularis. ${ }^{31}$ Alignments of P29 with these homologous proteins are shown in Figure 1.

\subsection{Cloning, expression and purification of $P 29$}

P29-amplified cDNA was directly inserted into the pET 151 $D /$ expression vector for the subsequent transformation of TOP 10 chemically competent $E$. coli. The cloning was verified by colony PCR. Among 10 selected positive colonies, six were shown to contain the plasmid with inserts in the correct orientation. The plasmids of one selected positive clone (no. 8 ) were transferred into the $E$. coli BL21 strain for expression. Expressed proteins were subsequently purified from bacterial lysate by affinity-purified cobalt chromatography, then identified on a 15\% Coomassie Blue-stained SDS-PAGE gel run under reducing conditions. The purified protein was stored at $-20^{\circ} \mathrm{C}$ until used for subsequent serological tests.

\subsection{Follow-up characteristics of recP29 ELISA}

The serological follow-up of patients from CCE and NCCE patients is shown in Figure 2. Among 40 CCE patients tested, $15(38 \%)$ showed no detectable anti-recP29 antibodies at the initial time of diagnosis, nor during the whole period of follow-up. The other 25 patients all showed anti-recP29 antibody response at different time points of follow-up. 

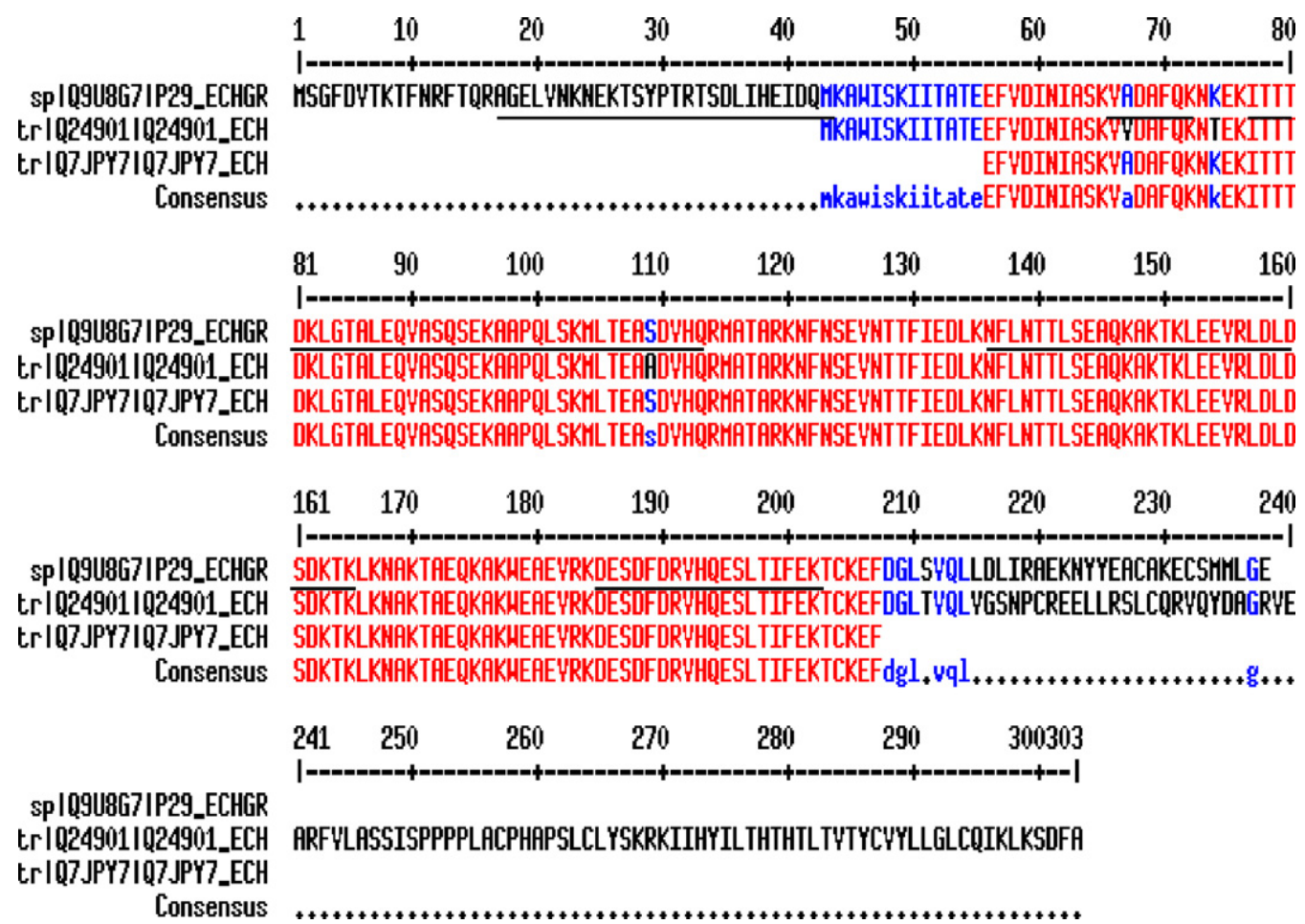

Figure 1 Alignment (MultAlin at the Institut National de Recherche Agronomique, Paris, France) of the deduced amino acid sequence of P29 (SwissProt accession no. Q9U8G7) with homologous proteins: antigen S epitope (Fragment) of Echinococcus granulosus (SwissProt accession no. Q7JPY7) and antigen 6 (Fragment) [Adama6] of E. multilocularis (SwissProt accession no. Q24901). Peptides identified by MS/MS are underlined.

In general, a progressive decrease of respective antibody levels during the timecourse of follow-up was observed. More precisely, at time of diagnosis (D0; before surgery), $32 \%$ of these patients exhibited a low but positive antibody level ( $A U<20)$, while the remaining, 30\% patients had no detectable anti-recP29 antibodies. Conversely, anti-HCF antibodies were initially detected in $86 \%$ of the patients, with generally high levels (AU 85-185). With regard to comparing hepatic versus pulmonary cyst localization, initial seronegativity by recP29 ELISA was seen in 12 out of 18 lung CCE cases and in all five lung NCCE cases, while two out of 11 liver CCE cases and two out of six liver NCCE cases were seronegative at the entrance into the study. Thus, the seronegativity rate was higher for lung localizations than for the liver. The post-treatment evolution of anti-recP29 antibody levels showed that in $80 \%$ of CCE patients (20 of 25 CCE cases), immunoglobulin levels increased during the first 1-6 months after surgery (including also those patients who were initially seronegative before treatment but who became seropositive after treatment), reaching their highest level at 1 month post-operation. Subsequently, levels started to progressively decrease, and anti-recP29 antibodies were detectable in only 15 and $11 \%$ at 1 and 2 years post-operation, respectively. Interestingly, no CCE patient showed any further anti-recP29 antibody reactivity past 3 years post-surgery, compared with $89 \%$ when using HCF as antigen (Table 3 ).

In general, recP29 ELISA showed a faster decrease of IgG levels than the HCF ELISA, and seronegativity was reached in all CCE patients $(100 \%)$ at the endpoint of follow-up of each

Table 3 Comparison of percentage seropositivity as determined by recP29 ELISA and HCF ELISA in 25 cured cystic echinococcosis CCE and 11 non-cured cystic echinococcosis (NCCE) patients during their follow-up period ${ }^{\text {. }}$.

\begin{tabular}{llllllll}
\hline & D0 & D7 & $1 M$ & $6 M$ & $1 Y$ & $2 Y$ & $\geq 3 Y$ \\
\hline CCE & $(n=22)$ & $(n=19)$ & $(n=22)$ & $(n=21)$ & $(n=20)$ & $(n=18)$ & $(n=19)$ \\
$\quad$ recP29 ELISA-positive (\%) & $7(32)$ & $15(79)$ & $18(82)$ & $6(29)$ & $3(15)$ & $2(11)$ & $0(0)$ \\
HCF ELISA-positive (\%) & $19(86)$ & $15(79)$ & $20(91)$ & $20(95)$ & $18(90)$ & $18(100)$ & $17(89)$ \\
NCCE & $(n=10)$ & $(n=7)$ & $(n=8)$ & $(n=10)$ & $(n=10)$ & $(n=6)$ & $(n=10)$ \\
recP29 ELISA-positive (\%) & $3(30)$ & $5(71.4)$ & $7(87.5)$ & $8(80)$ & $7(70)$ & $5(83.3)$ & $7(70)$ \\
HCF ELISA-positive (\%) & $8(80)$ & $7(100)$ & $8(100)$ & $9(90)$ & $9(90)$ & $6(100)$ & $10(100)$ \\
\hline
\end{tabular}

a Serum samples were collected before surgery (D0) and 1 week (D7), 1 month (1 M), 6 months (6M), 1 year (1Y), 2 years (2Y) and 3 or more years $(\geq 3 Y)$ after surgery. Statistically significant differences between both clinical groups was noticed in recP29 ELISA $(P<0.05)$. 

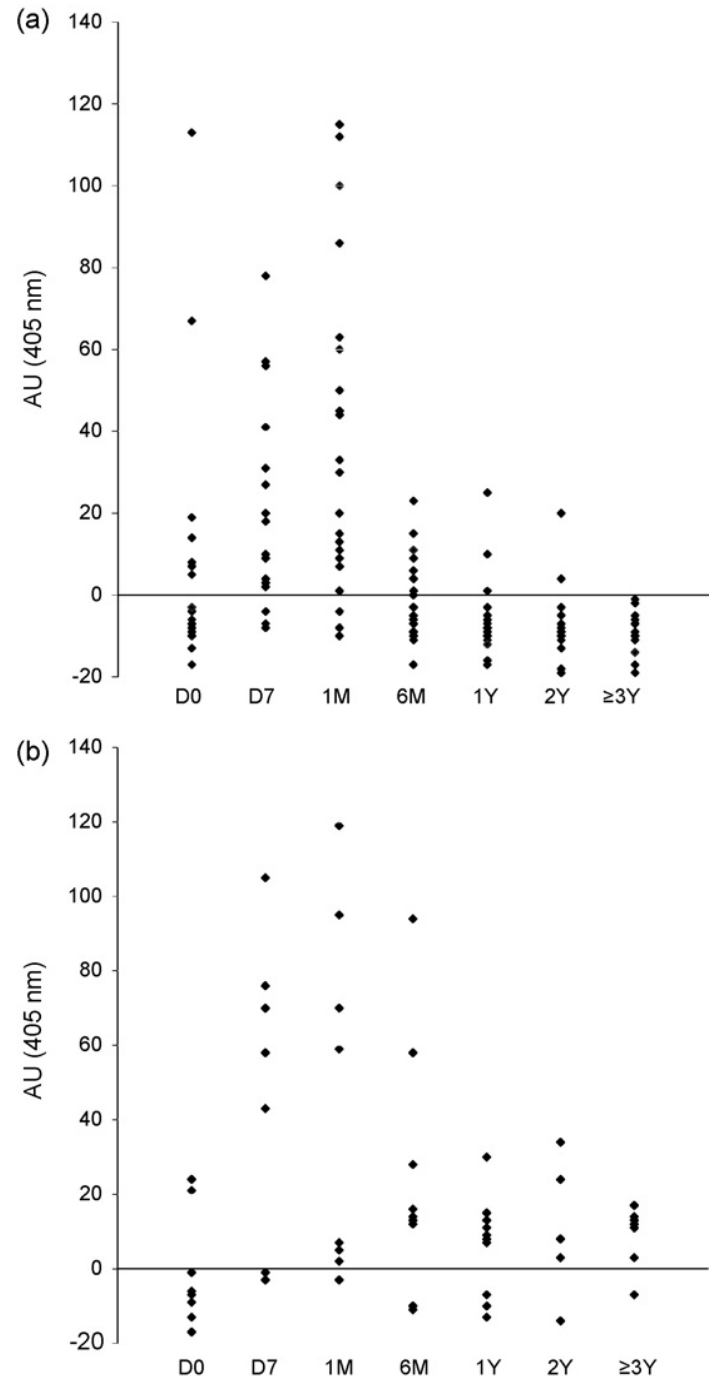

Figure 2 Post-treatment evolution of antibody levels expressed as antibody units (AU), determined by ELISA in 29 cured cystic echinococcosis (A) and 11 non-cured cystic echinococcosis (B) patients. Serum samples were collected before surgery (D0) and 1 week (D7), 1 month (1M), 6 months $(6 \mathrm{M}), 1$ year $(1 \mathrm{Y}), 2$ years $(2 \mathrm{Y})$ and 3 or more years $(\geq 3 \mathrm{Y})$ after surgery.

patient (which ranged between 1 and 5 years post-surgery), compared with only $10 \%$ as determined with the HCF ELISA. Some sera were followed-up for another few years, although seronegativity had already been reached at 1, 2 and 3 years after treatment. However, recP29 ELISA of 14 patients with still-active CE (NCCE) showed that three of them (21\%) had no detectable anti-recP29 antibodies at time of diagnosis (D0) and also during the whole period of follow-up. At DO (before surgery), 30\% from the remaining 11 patients who were initially positive (with generally low antibody levels of $A U<24$ ) reached an increasing maximum level at 1 month post-surgery (Table 3). Subsequently, a progressive decrease but without seronegativity at the endpoint of follow-up was observed, except for one patient, who became seronegative within 3 years after surgery and remained negative for the subsequent 4 and 5 years after surgery, while the patient was clinically considered to have still active cysts with small daughter vesicles. Overall, 10 out of 11 NCCE (91\%) patients remained positive at the endpoint of followup ( $1-5$ years post-surgery). HCF ELISA with NCCE patients exhibited higher antibody levels as well as higher rates of seropositivity than recP29 ELISA at diagnosis and during the whole follow-up period. None of the patients became negative at the endpoint of follow-up. A comparative analysis of sensitivities regarding recP29 and HCF in CCE and NCCE patients is shown in Table 3.

\subsection{Follow-up characteristics of recP29 immunoblot}

The same cohort of follow-up sera collected from CCE and NCCE patients were also tested by immunoblotting for their immunoreactivity to recP29 (Figures 3 and 4). Of 40 CCE patients, 11 (28\%) were serologically negative to recP29 at D0 and during the whole period of follow-up; the remaining 29 patients were immunoreactive to the recombinant protein. With regard to comparing hepatic versus pulmonary cyst localization, initial seronegativity by recP29 immunoblot was seen in nine out of 18 lung CCE cases and three out of five lung NCCE cases, while one out of 11 liver CCE cases and three out of six liver NCCE cases were seronegative at the entrance into the study. Thus, the seronegativity rate was higher for lung localizations than for the liver. These 29 patients immunoreactive to recP29 (including also
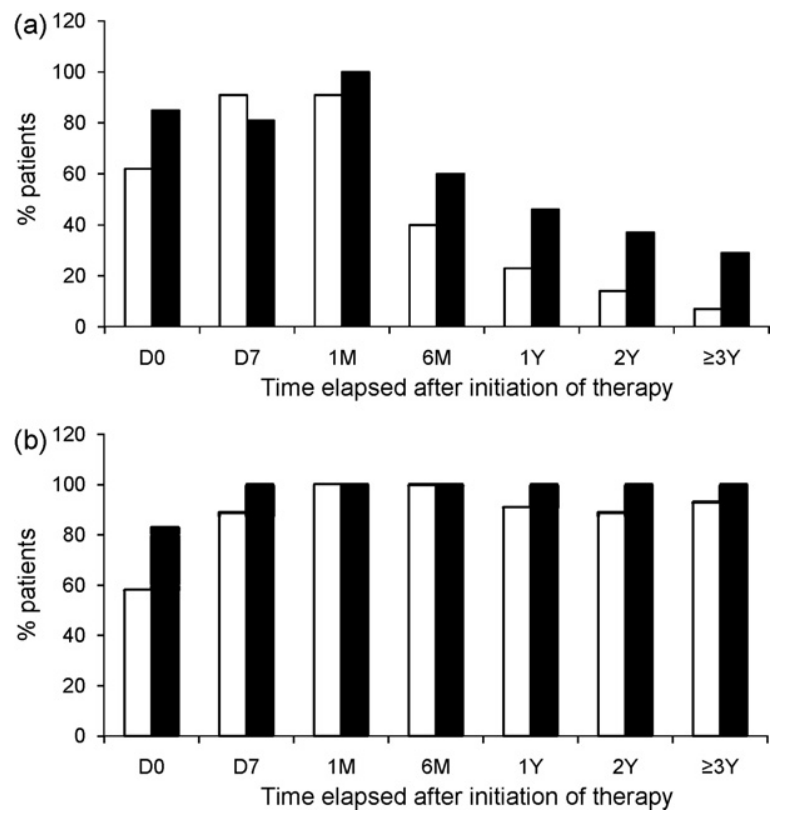

Figure 3 Comparison of temporal evolution between recP29 band (white) recognition and hydatid cyst fluid (AgB) (black) by immunoblotting in 29 cured cystic echinococcosis $(A)$ and 11 non-cured cystic echinococcosis (B) patients upon timecourse after treatment. Serum samples were collected before surgery (D0) and 1 week (D7), 1 month (1M), 6 months $(6 \mathrm{M})$, 1 year (1Y) and 3 or more years $(\geq 3 Y)$ after surgery. Statistically significant differences between the two clinical groups was observed with both recP29 immunoblotting and HCF immunoblotting $(P=0.012 ; P=0.005)$. 

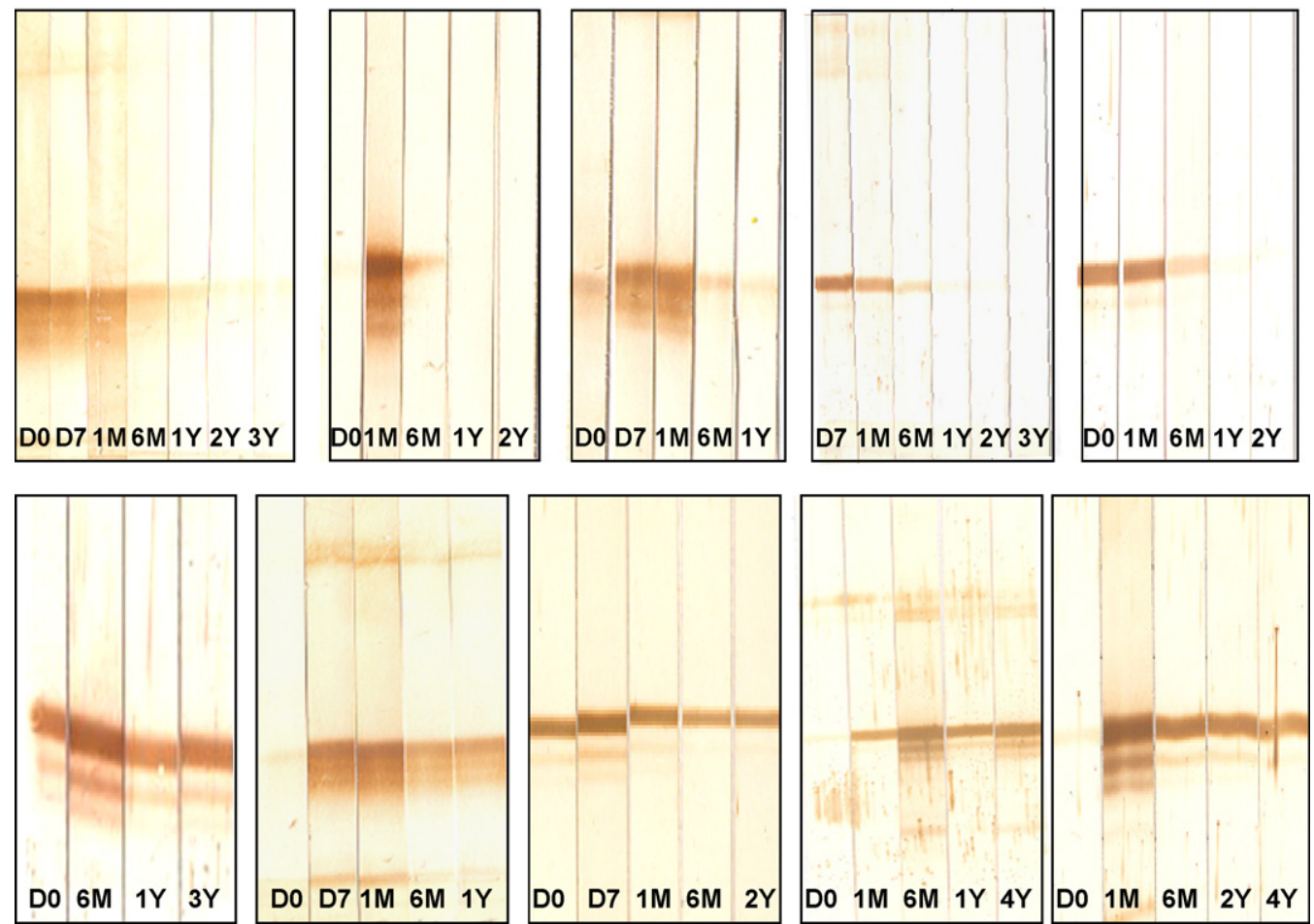

Figure 4 Representative examples of immunoblotting profiles using recP29 as antigen, and shown with an example of five cured cystic echinococcosis (A) and five non-cured cystic echinococcosis (B) patients whose sera were collected before surgery (D0) and 1 week (D7), 1 month $(1 \mathrm{M}), 6$ months $(6 \mathrm{M}), 1$ year (1Y), 2 years $(2 \mathrm{Y}), 3$ years (3Y) and 4 years $(4 \mathrm{Y})$ after surgery.

those patients who were initially seronegative before treatment but who became seropositive after treatment) showed an interesting progressive decrease of anti-recP29 banding intensity. This effect was mostly noticed at 6 months postsurgery, when the protein was recognized in $40 \%$ of patients (detectability rate among 29 patients). At later time points, seropositivity sharply decreased to $7 \%$ over 3 years posttreatment, compared to $29 \%$ in HCF immunoblot (Figure 3A). All patients $(n=29)$ converted to negative at their respective endpoint of follow-up except two, who remained positive at 1 and 2 years post-surgery, respectively. Nevertheless, all of them were negative by recP29 ELISA but remained still positive in the HCF ELISA.

Thus, the overall immunoblot negativity at the endpoint of the follow-up period was documented in 27 out of 29 patients (93\%), compared with $72 \%$ (21 out of 29 patients) obtained by HCF immunoblot. In general, CCE patients exhibited a faster progressive decrease of immunoreactivity as well as higher rate of seronegativity to recP29 in immunoblotting than observed with HCF immunoblot (antibody reactivity to AgB fractions). A direct comparison between seroreactivities to both antigens is shown in Figure 3A. Conversely, all 14 NCCE patients showed a stronger recognition of recP29, which persisted for years post-surgery until the endpoint of follow-up, except one NCCE patient, who showed a weak anti-recP29 reactivity at 3, 4 and 5 years post-treatment. This patient converted also to negative in the recP29 ELISA. Overall, recP29 was persistently recognized in 13 out of 14 NCCE patients (93\%) over 3 years post-surgery, compared with $100 \%$ when tested with HCF (AgB) immunoblotting. Statistically significant differences between CCE and NCCE patients were noticed in both recP29 ELISA and recP29 immunoblotting $(P=0.029$, $P=0.012)$ and with HCF-immunoblotting $(P=0.005)$.

A comparison between CCE patients that obtained either surgical treatment alone or surgery plus albendazole (ABZ) therapy showed no difference at the descriptive level (the patients' numbers being too small to allow a proper statistical analysis). The same holds true for the comparison between NCCE patients with either surgery alone, ABZ alone or surgery plus $A B Z$, where no differences became apparent (data not shown). More patients need to be respectively investigated in order to reach statistical significance for such analyses.

\section{Discussion}

Two native $E$. granulosus protoscolex-specific 'bands' identified in a previous study ${ }^{23}$ were subjected to MS/MS analysis, yielding high sequence homologies with either the P29 protein $^{29}$ or the 14-3-3 isoform 2 of E. granulosus. ${ }^{32}$ Based on the biology of 14-3-3, we did not see any reason to include this antigen as a potential candidate for the present serological follow-up study. Conversely, the P29 protein first described by Gonzalez et al. ${ }^{29}$ appeared interesting by its expression being restricted to $E$. granulosus protoscoleces ${ }^{33}$ and by exhibiting a good immunodiagnostic potential to detect CE in patients before surgery. ${ }^{29}$ Our investigations now showed that a recombinant P29 also exhibits a good prognostic value in the follow-up monitoring of those cured versus non-cured CE patients who were able to develop a 
seropositive status against recP29 either before or shortly after diagnosis and/or initiation of a treatment protocol, respectively. A certain portion of CE patients remained constantly negative against recP29 from day 0 (before surgery) until the endpoint of the present follow-up (38\% CCE and $21 \%$ of NCCE patients for ELISA, and $27 \%$ of CCE patients only for immunoblotting). This could be related to the observation that most of these anti-recP29-negative patients presented lung cysts. It is commonly known that lung cysts do not yield an intense humoral response due to separation of the cysts from the surrounding pulmonal tissue and therefore weak release of parasite metabolites (antigens) into the pulmonal circulation, resulting in a poor host immune response. In addition, lung cysts are frequently sterile; therefore even after a putative rupture or otherwise exposure to cyst content, there would have been no protoscolex-dependent antigenic challenge. As P29 appears restricted, in its expression, to protoscoleces, this would then result in a lack of P29-stimulation and consequently in a respective seronegativity. Moreover, some of the CE patients who were seronegative to recP29 at D0 (before surgical intervention) seroconverted after treatment and demonstrated increasing anti-recP29 antibody levels during the first 6 months after surgery. This may have indicated a post-operative protoscolex-exposure; in that case, antirecP29 serology may be of interesting prognostic value in the follow-up of CE patients. When comparing recP29 by ELISA and immunoblotting, it became evident that ELISA showed a lower diagnostic and post-operative sensitivity than immunoblotting, especially also in the frame of longterm follow-up assessments. Anti-recP29 seronegativity was reached more quickly by ELISA than by immunoblotting, i.e. all initially anti-recP29-reactive CCE patients had negative ELISA results at latest by 3 years post-surgery. A possible reason for this may be the structural difference of the antigen, as in ELISA the recombinant protein remains more or less native, with some of the conformational epitopes being kept obscured. In immunoblotting, however, most proteins become linearized and thus potentially hide epitopes for respective antibody-binding capacities.

We also directly compared the prognostic value of the recP29 antigen with that of native HCF. In the recP29 ELISA, seronegativity in CCE patients was reached much faster than in HCF ELISA. While all CCE patients who initially had an antirecP29 seropositivity became anti-recP29-negative within 3 years after surgery, $86 \%$ of corresponding CCE patients were still HCF-ELISA-positive more than 3 years after operation. Furthermore, HCF ELISA did not allow the detection of differences in the post-operative antibody level development between CCE and NCCE patients (differences were statistically not significant; $P>0.05$ ).

$\mathrm{Li}$ et al. ${ }^{16}$ reported a recombinant protein (termed 'EpC1') that was expressed from a protoscolex cDNA library. They showed a slightly higher (92\%) sensitivity than that obtained with HCF at DO (before surgery). By contrast to our recP29, however, EpC1 immunoblotting did not decrease during follow-up after treatment and was still high (92\%) even with sera obtained 6 years after surgery.

Sequence analyses showed that the P29 gene presents 97\% homology to the sequence of Antigen 6 (fragment Adama 6) of $E$. multilocularis. ${ }^{31}$ Therefore, we can assume that sera from $E$. multilocularis patients may exhibit a cross-reactive potential with recP29, and we will address this point in future investigations.

P29 also showed full identity (100\%) with the Antigen ' $S$ ' epitope (Fragment) of $E$. granulosus ${ }^{30}$ at the amino acid level. The Ag 'S' epitope (152 amino acids) was characterized as an immunogenic epitope responsible for precipitation of 'arc 5' in immunoelectrophoresis. ${ }^{34}$ Immunological and proteomic analyses by peptide fingerprinting on the Ag5 and P29 relationship showed that P29 and Ag5 are immunologically related but are distinct proteins. ${ }^{29}$ Chamekh et al. ${ }^{22}$ reported a synthetic peptide of 34 amino acids, designated 89-120, which mimics an epitope of Ag5; this peptide showed a high diagnostic performance with regard to sensitivity and specificity. ${ }^{35}$ This peptide corresponds also to the region involved in cross-reactivity with P29 (residues 144-177 of P29) with Ag 5. ${ }^{29}$ We suggest that more work needs to be done to further elucidate the biological and genetic relationship between antigen 6, antigen 5 and P29.

In conclusion, we have successfully cloned and expressed the gene encoding for P29, a protoscolex-specific component of $E$. granulosus. Our preliminary results showed that recP29 demonstrated a very good performance as an antigen marker for the serological follow-up of human CE in those patients who were capable of mounting an anti-recP29 antibody response either before shortly after diagnosis and/or initiation of treatment. We suggest that recP29 serology should be more intensively tested with CE follow-up cases of other ages and other geographical areas in order to provide a more extensive documentation of the prognostic capacity of this tool.

Authors' contributions: BG, NM, HB and NBN designed the study and drafted the manuscript; NBN performed the laboratory work and interpretation of data; CG contributed to the molecular and cloning part of the work; MG contributed to the serological part of the work; AN provided all clinical and histological information on the patients. All authors read and approved the final manuscript. BG is guarantor of the paper.

Acknowledgements: The authors would like to acknowledge Elisabeth Frei and Christine Wittwer for their support in laboratory methodology.

Funding: This work was supported by the Swiss National Science Foundation (grant no. 31-111780/1), by the HansSigrist Foundation (grant no. 31-111780/1) and by the Tunisian High Education Ministry (grant no. 65/05).

Conflicts of interest: None declared.

Ethical approval: Not required.

\section{References}

1. Pawlowski ZS, Eckert J, Vuitton DA, Ammann RW, Kern P, Craig PS, et al. Echinococcosis in humans: clinical aspects, diagnosis and treatment. In: Eckert J, Gemmel MA, Meslin FX, Pawlowski ZS, editors. WHO/OIE manual on echinococcosis in humans and 
animals: public health problem of global concern. Paris: World Organization for Animal Health; 2001. p. 20-66.

2. Wen H, New RR, Craig PS. Diagnosis and treatment of human hydatidosis. Brit J Clin Pharmacol 1993;35:565-74.

3. Doiz O, Benito R, Gil A, Rojas J, Rubio MC, Gomez Lus R. Pre-and post-surgical detection of IgG, IgM and IgA specific to hydatidosis by ELISA with purified antigen enriched with the $5 / \mathrm{B}$ antigen complex. J Clin Lab Anal 2002;16:295-8.

4. Gottstein B. Molecular and immunological diagnosis of echinococcosis. Clin Microbiol Rev 1992;5:248-61.

5. Lawn SD, Bligh J, Craig PS, Chiodini PL. Human cystic echinococosis: evaluation of post-treatment serologic followup by IgG subclass antibody detection. Am J Trop Med Hyg 2004;70:329-35.

6. Lightowlers MW, Gottstein B. Echinococcosis/hydatidosis: antigens, immunological and molecular diagnosis. In: Thompson $\mathrm{RCA}$, Lymbery AJ, editors. Echinococcus and hydatid disease. Oxford: CAB International; 1995.

7. Poretti D, Felleisen E, Grimm F, Pfister M, Teuscher F, Zuercher $C$, et al. Differential immunodiagnosis of human cystic hydatid disease and other cross-reactive pathologies. Am J Trop Med Hyg 1999;60:193-8.

8. Zhang W, Li J, MCManus DP. Concepts in immunology and diagnosis of hydatid disease. Clin Microbiol Rev 2003;16:18-36.

9. Ortona E, Rigano R, Margutti P, Notargiacomo S, loppolo S, Vaccari $S$, et al. Native and recombinant antigens in the immunodiagnosis of human cystic echinococcosis. Parasite Immunol 2000;22:553-9.

10. Ben Nouir N, Nuñez S, Frei E, Gorcii M, Müller N, Nouri A, et al. Post-surgical follow-up (by ELISA and immunoblotting) of cured versus non-cured cystic echinococcosis in young patients. Parasitol 2008;135:105-14.

11. Craig PS, Zeyhle E, Romig T. Hydatid disease research and control in Turkana II. The role of the immunological techniques for the diagnosis of hydatid disease. Trans $R$ Soc Trop Med Hyg 1986;80:183-92.

12. Gottstein B, Eckert J, Woodtli W. Determination of parasitespecific immunoglobulines using the ELISA in patients with echinococcosis treated with mebendazole. Z Parasitenkd 1984;70:385-9.

13. Fernandez V, Fereira HB, Fernández C, Zaha A, Nieto A. Molecular characterization of a novel 8-kDa subunit of Echinococcus granulosus antigen B. Mol Biochem Parasitol 1996;77:247-50.

14. Rott MB, Fernández V, Farias $S$, Ceni J, Ferreira HB, Haag $\mathrm{KL}$, et al. Comparative analysis of two different subunits of antigen B from Echinococcus granulosus: gene sequences, expression in Escherichia coli and serological evaluation. Acta Trop 2000;75:331-40.

15. Lorenzo C, Last JA, González-Sapienza GG. The immunogenicity of Echinococcus granulosus antigen 5 is determined by its post-translational modifications. Parasitol 2005;131:669-77.

16. Li J, Zhang WB, Wilson M, Ito A, McManus D. A novel recombinant antigen for immunodiagnosis of human cystic echinococcosis. J Infect Dis 2003;188:1951-60.

17. Virginio VG, Hernández A, Rott MB, Monteiro KM, Zandonai $A F$, Nieto $A$, et al. A set of recombinant antigens from Echinococcus granulosus with potential for use in the immunodiagnosis of human cystic hydatid disease. Clin Exp Immunol 2003;132:309-15.

18. Li J, Zhang WB, Wilson M, Ito A, MCManus DP. Recombinant antigens for immunodiagnosis of cystic echinococcosis. Biol Proc 2004;6:67-77.

19. Mamuti W, Yamasaki H, Sako Y, Nakao MM, Xiao N, Nakaya K, et al. Molecular cloning, expression, and serological evaluation of an 8-kilodalton subunit of antigen B from Echinococcus multilocularis. J Clin Microbiol 2004;42:1082-8.
20. Gonzales-Sapienza G, Lorenzo C, Nieto A. Improved immunodiagnosis of cystic hydatid disease by using a synthetic peptide with higher diagnostic value than that of its parent protein. Echinococcus granulosus antigen B J Clin Microbiol 2000;38:3979-83.

21. Lorenzo C, Ferreira HB, Monteiro KM, Rosenzvit M, Kamenetzky L, Garcíaet HH, et al. Comparative analysis of the diagnostic performance of six major Echinococcus granulosus antigens assessed in a double-blind randomized multicenter study. J Clin Microbiol 2005;43:2764-70.

22. Chamekh M, Gras Masse H, Bossus M, Facon B, Dissous C, Tartar $A$, et al. Diagnostic value of a synthetic peptide derived from Echinococcus granulosus recombinant protein. J Clin Invest 1992;89:458-64.

23. Ben Nouir N, Nuňez S, Gianinazzi C, Gorcii M, Müller N, Nouri A, et al. Assessment of Echinococcus granulosus somatic protoscolex antigens for the serological follow-up of young patients with surgically treated cystic echinococcosis. J Clin Microbiol 2008;46:1631-40.

24. Gottstein B, Schantz PM, Todorov T, Saimot AG, Jacquier P. An international study of the serological differential diagnosis of human cystic and alveolar echinococcosis. Bull World Health Organ 1986;64:101-5.

25. Shevchenko A, Wilm M, Vorm O, Mann M. Mass spectrometric sequencing of proteins silver-stained polyacrylamide gels. Anal Chem 1996;68:850-8.

26. World Health Organization Informal Working Group. International classification of ultrasound images in cystic echinococcosis for application in clinical and field epidemiological settings. Acta Trop 2003;85:253-61.

27. Gottstein B, Jacquier P, Bresson-Hadni S, Eckert J. Improved primary immunodiagnosis of alveolar echinococcosis in humans by an enzyme-linked immunosorbent assay using the $\mathrm{Em}^{\text {plus }}$. antigen. J Clin Microbiol 1993;31:373-6.

28. Siles-Lucas M, Gottstein B. The 14-3-3 protein: a key molecule in parasites as in other organisms. Trends Parasitol 2003;19:575-81.

29. Gonzalez G, Spinelli P, Lorenzo C, Hellman U, Nieto A, Willis A, et al. Molecular characterization of p-29 a metacestode specific component of Echinococcus granulosus which is immunologically related to but distinct from antigen 5 . Mol Biochem Parasitol 2000;105:177-84.

30. Facon B, Chamekh M, Dissous C, Capron A. Molecular cloning of an Echinococcus granulosus protein expressing an immunogenic epitope of antigen 5. Mol Biochem Parasitol 1991;45: 233-9.

31. Siles-Lucas M, Gottstein B, Felleisen R. Identification of differentially expressed Echinococcus multilocularis Em 6 potentially related to antigen 5 of Echinococcus granulosus. Parasite Immunol 1998;20:473-81.

32. Siles-Lucas $M$, Nunes CP, Zaha A, Breijo M. The 14-3-3 protein is secreted by the adult worm of Echinococcus granulosus. Parasite Immunol 2000;22:521-8.

33. Chemale G, Van Rossum AJ, Jefferies JR, Barrett J, Brophy PM, Ferreira HB, et al. Proteomic analysis of the larval stage of the parasite Echinococcus granulosus: causative agent of cystic hydatid disease. Proteomics 2003;3:1633-6.

34. Capron A, Vernes A, Biguet J. Le diagnostic immunoéléctrophorétique de l'hydatidose. In: Le kyste hydatique du foie. Lyon: SIMEP; 1967. p. 27-40.

35. Barbieri M, Fernández V, González G, Luaces VM, Nieto A. Diagnostic evaluation of a synthetic peptide derived from a novel antigen $B$ subunit as related to other available peptides and native antigens used for serology of cystic hydatidosis. Parasite Immunol 1998;20:51-61. 